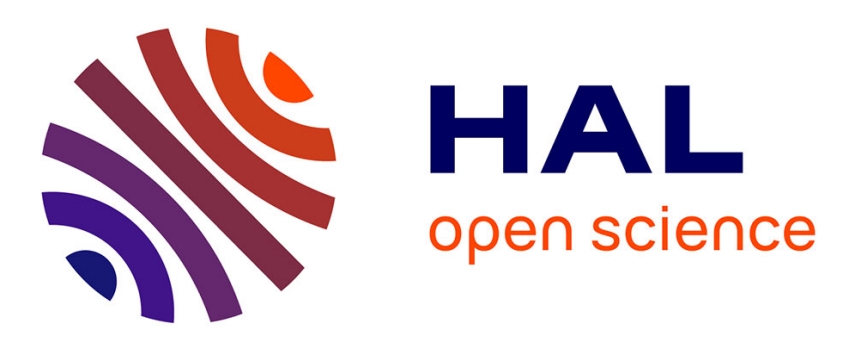

\title{
Enhanced membrane rigidity in charged lamellar phases
}

Paul G. Higgs, Jean-François Joanny

\section{- To cite this version:}

Paul G. Higgs, Jean-François Joanny. Enhanced membrane rigidity in charged lamellar phases. Journal de Physique, 1990, 51 (20), pp.2307-2320. 10.1051/jphys:0199000510200230700 . jpa-00212530

\section{HAL Id: jpa-00212530 https://hal.science/jpa-00212530}

Submitted on 1 Jan 1990

HAL is a multi-disciplinary open access archive for the deposit and dissemination of scientific research documents, whether they are published or not. The documents may come from teaching and research institutions in France or abroad, or from public or private research centers.
L'archive ouverte pluridisciplinaire HAL, est destinée au dépôt et à la diffusion de documents scientifiques de niveau recherche, publiés ou non, émanant des établissements d'enseignement et de recherche français ou étrangers, des laboratoires publics ou privés. 
Classification

Physics Abstracts

$61.30-68.10-82.70$

\title{
Enhanced membrane rigidity in charged lamellar phases
}

\author{
Paul G. Higgs and Jean-François Joanny \\ C.N.R.S., Institut Charles Sadron, 6 rue Boussingault, 67083 Strasbourg, France
}

(Received 20 April 1990, accepted in final form 28 June 1990)

\begin{abstract}
Résumé. - Nous calculons la contribution électrostatique à l'énergie de courbure de membranes de tensioactifs ioniques dans des phases lamellaires quand les interactions électrostatiques ne sont pas écrantées par du sel. Dans la limite des fortes densités de charge (quand la longueur de GouyChapmann $\lambda$ est plus petite que la distance entre membranes $d$ ) nous montrons que l'énergie de courbure est proportionnelle à la distance interlamellaire : $k_{\mathrm{c}} \sim \frac{d}{\ell} k_{\mathrm{B}} T$ où $\ell$ est la longueur de Bjerrum. Ce calcul est fait à la fois à partir de l'énergie libre de deux membranes ondulant de façon symétrique et à partir de l'énergie libre de deux membranes cylindriques concentriques. La valeur de l'énergie de courbure est grande par rapport à $k_{B} T$, les membranes sont donc plates et rigides. Nous considérons aussi le cas des faibles densités de charge $(\lambda>d)$ et le cas d'interactions écrantées avec une longueur de Debye $\kappa^{-1}>d$ et nous montrons que l'énergie de courbure ne suit pas la même loi d'échelle pour des surfaces ondulées et dans la géométrie cylindrique. Nous donnons la valeur de $k_{\mathrm{c}}$ dans toutes ces limites.
\end{abstract}

\begin{abstract}
We calculate the electrostatic contribution to the bending energy of charged surfactant membranes in lamellar phases when the electrostatic interactions are not screened by added salt. In the limit of high charge densities (when the Gouy-Chapman length $\lambda$ is smaller than the membrane separation $d$ ) we find that the bending constant is proportional to the intermembrane spacing: $k_{\mathrm{c}} \sim \frac{d}{\ell} k_{\mathrm{B}} T$, where $\ell$ is the Bjerrum length. This is calculated both from the electrostatic free energy of two membranes undulating symmetrically and from the free energy of two concentric cylindrical membranes. The value of the bending energy is potentially very large compared to $k_{\mathrm{B}} T$, hence the membranes are extremely flat and rigid. We also consider the case of small charge densities $(\lambda>d)$, and the case of screened interactions with the Debye length $\kappa^{-1}>d$, and show that the bending energy does not scale in the same way for the undulating surface and the cylindrical geometry. We give the scaling behavior of $k_{\mathrm{c}}$ in all these limits.
\end{abstract}

\section{Introduction.}

Lamellar phases formed by amphiphilic molecules consist of a stack of alternating solvent layers and surfactant membranes. The stability of the structure is governed by the interactions between neighbouring membranes mediated by the solvent layers, these interactions include 
the Van der Waals attractive forces, the hydration forces and the electrostatic forces, when present.

It was argued by Helfrich [1] that due to the very low surface tensions, the out-of-plane undulations of the membranes are important and induce a repulsion of entropic origin between them. The strength of the undulation repulsion is controlled by the bending elasticity of the membranes that is usually described by the bending constants $k_{\mathrm{c}}$ and $\bar{k}_{\mathrm{c}}$ for the mean and Gaussian curvatures.

For neutral surfactants the bending energy arises from the rearrangement of the molecular packing when the membrane is curved. The bending constants are typically of order the thermal energy $k_{\mathrm{B}} T$ and can be reduced by adding a cosurfactant. In organic solvents, if $k_{\mathrm{c}}$ is small enough, the repulsive undulation repulsion may dominate the Van der Waals attraction and stabilize the lamellar structure up to very large intermembrane spacings.

For charged surfactant membranes in water, the repulsive electrostatic interactions contribute to the stability of the structure. They also reduce the out-of-plane fluctuations and thus give a positive contribution to the bending constant which in some cases may be much larger than the intrinsic contribution. This reduces then considerably the undulation repulsion.

In order to study the role of the electrostatic interactions, Pincus et al. [2] have identified three important length scales in the problem :

i) the mean membrane separation in the lamellar phase $2 d$;

ii) the Debye screening length $\kappa^{-1}=\left(8 \pi n_{\infty} \ell\right)^{-1 / 2}$;

iii) the Gouy-Chapman length $\lambda=e /(2 \pi \ell \sigma)$;

where $n_{\infty}$ is the bulk electrolyte concentration, $\sigma$ is the surface charge density on one side of the membrane, and $\ell=e^{2} /(4 \pi \varepsilon T)$ is the Bjerrum length. We omit Boltzmann's constant from now on and choose temperature units where it is equal to one.

Winterhalter and Helfrich [3] have studied the limit of high salt concentrations, where the screening length $\kappa^{-1}$ is the smallest length in the problem $(\kappa d>1, \kappa \lambda>1)$. The linearised Debye-Hückel equation is valid for the potential. The electrostatic contributions to the bending constants are evaluated by comparison of the energy of cylindrically and spherically shaped membranes with flat planes. It is found that

$$
k_{\mathrm{c}}=\frac{3 \sigma^{2}}{4 \varepsilon \kappa^{3}} \sim \frac{T}{\kappa^{3} \ell \lambda^{2}}, \quad(\kappa d>1, \kappa \lambda>1) .
$$

Duplantier, Goldstein et al. [4, 5] and Bensimon et al. [6] have shown that the same result applies if the potential is calculated about an undulating surface. Pincus et al. [2] have shown that the linearised equation is also valid if $\kappa d<1$ provided that $\kappa^{2} \lambda d>1$. In this case

$$
k_{\mathrm{c}} \sim \frac{T}{\kappa^{3} \ell \lambda^{2}(\kappa d)^{3}}, \quad\left(\kappa d<1, \kappa^{2} \lambda d>1\right)
$$

which has a smooth crossover to (1) when $\kappa d=1$, as required. We plot these different regions in figure 1 using axes $\kappa^{-1}$ and $d$ for a fixed value of $\lambda$ (this is analogous to the figure of reference [2] where $d$ is fixed).

If $\kappa d>1$ but $\kappa \lambda<1$ we are in the "intermediate" regime of reference [2] where the Debye-Hückel theory is no longer valid and the non-linear Poisson-Boltzmann equation must be solved. This has been done by Mitchell and Ninham [7] and Lekkerkerker [8], and gives

$$
k_{\mathrm{c}}=\frac{T}{\pi \kappa \ell}, \quad(\kappa d>1, \kappa \lambda<1) .
$$




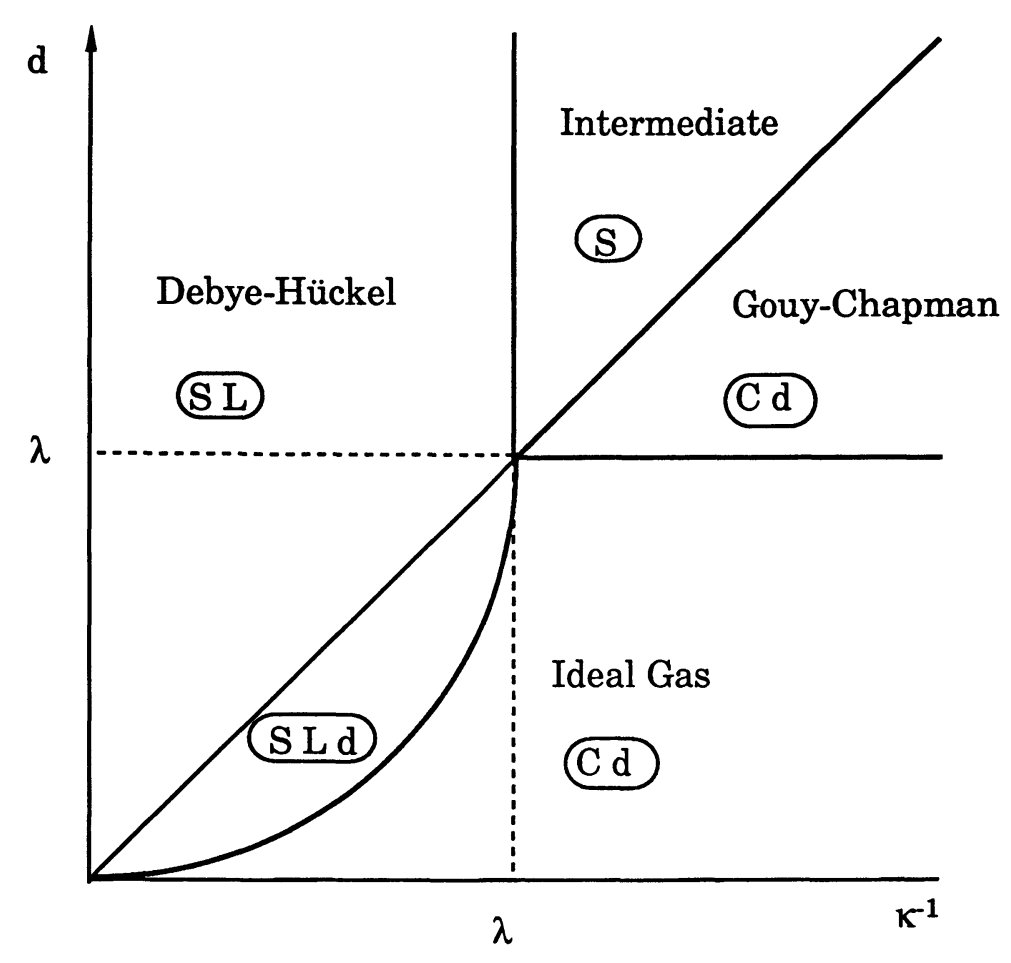

Fig. 1. - Boundaries between the regions of validity for the different solutions of the problem shown as functions of membrane separation and screening length for a fixed value of S - Salt important, C Counterions important, L - Linearized equation valid, $d$ - Membrane separation important.

For the two other regions discussed by Pincus et al. [2] no exact solution for the bending constant has been found, but the scaling behaviour with the different lengths has been obtained, both by arguments of continuity with the regions of known solution, and by considering the cylindrical geometry described below. In this article we consider the GouyChapman region in the limit of high surface charge and zero salt concentration: $\lambda<d<\kappa^{-1}$. We show explicitly that the result conjectured in [2] is correct for both the undulating surface geometry and the cylindrical geometry. For the ideal gas region which has negligible salt concentration and low surface charge $\left(d<\lambda<\kappa^{-1}\right)$ we present further arguments in support of the conjectures of [2]. We find, however, that for the ideal gas region and for the Debye-Hückel region with $\kappa d<1$, in both of which cases $d$ is the smallest length in the problem, the cylindrical and undulating surface geometries do not give the same result. This is discussed further in section 3.

A summary of the different regions and the predicted scaling of the bending constant is presented in table I.

\section{The Gouy-Chapman region.}

We consider limit of high surface charge where the Gouy-Chapman length $\lambda$ is smaller than the spacing $d$ and the added salt concentration is negligible. We note that $d$ is still important in this case since it is the finite value of $d$ which imposes the finite concentration of counterions. The corresponding Debye-Hückel case with $\kappa^{-1} \ll d$ becomes a one-membrane problem since there is a finite salt concentration independent of $d$. 
Table I. - Summary of the regions of validity for the different approximations used, and predictions of the scaling behaviour of the bending constant.

\begin{tabular}{lcc} 
Region of approximation & \multicolumn{1}{c}{ Conditions } & Scaling of $k_{\mathrm{c}} / k_{\mathrm{B}} T$ \\
Debye-Hückel (large $d$ ) & $\kappa d>1, \kappa \lambda>1$ & $\frac{1}{\kappa^{3} \ell \lambda^{2}}$ \\
Debye-Hückel (small $d$ ) & $\kappa d<1, \kappa^{2} \lambda d>1$ & $\frac{1}{\kappa^{3} \ell \lambda^{2}(\kappa d)^{3}}$ \\
Intermediate & $\kappa d>1, k \lambda<1$ & $\frac{1}{\kappa \ell}$ \\
Gouy-Chapman & $\lambda<d<\kappa^{-1}$ & $\frac{d}{\ell}$ \\
Ideal gas & $d<\lambda<\kappa^{-1}$ & $\frac{\lambda}{\ell}$
\end{tabular}

We therefore wish to find the potential between two membranes which we take to be curved with a displacement from their mean position $u(x)=u \cos q x$, with a small amplitude $u \ll d$. For simplicity we consider the two membranes to have opposite displacements so that there is a plane of symmetry between the two as sketched in figure 2 . The electrostatic potential satisfies the Poisson-Boltzmann equation

$$
\nabla^{2} \phi=-\frac{n_{0} e}{\varepsilon} \exp \left(-\frac{e \phi}{T}\right)
$$

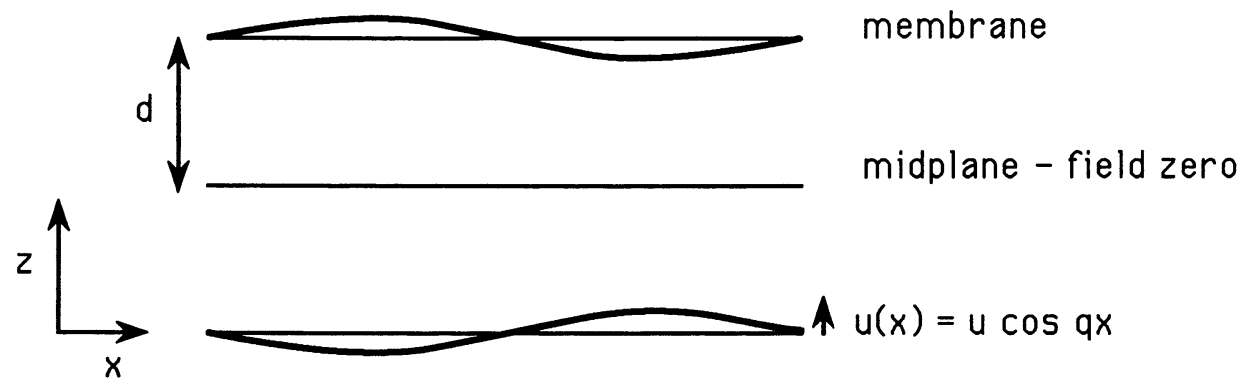

Fig. 2. - Undulating surface geometry. We consider the two membranes with oscillations of opposite phase.

We assume that there is a fixed (negative) surface charge - $\sigma$ on each side of the membranes so that at the surface the normal component of the field is $+\sigma / \varepsilon$. Due to symmetry the $z$ component of the field is zero at $z=d$. These boundary conditions assume the complete independence of the two sides of the membrane, and lead to a potential difference across it when it is curved. For this to be valid the energy stored in the field within the membranes must be much smaller than the curvature energy calculated from the field between the membranes. The condition for the validity of these boundary conditions (to be 
demonstrated in Appendix B) is

$$
t>\frac{\varepsilon_{\mathrm{L}}}{\varepsilon} d
$$

where $t$ is the membrane thickness, $\varepsilon_{\mathrm{L}}$ is the permittivity of the lipid within the membranes, and $\varepsilon$ is that of water (typically 40 times larger). We are not interested in corrections due to the membrane thickness, but we do not take the strict limit $t=0$. This would correspond to a single layer of charge density $2 \sigma$ and has a slightly different behavior. The same point arises in the Debye-Hückel case, and is discussed by Kiometzis and Kleinert [9]. These authors obtain a general result for $k_{\mathrm{c}}$ as a function of $\varepsilon_{\mathrm{L}}$ and $t$ from which both of the limits discussed above may be obtained. The equivalent condition to (5) is then $t>\varepsilon_{\mathrm{L}} / \varepsilon \kappa$, which is also given by Winterhalter and Helfrich [3].

In order to calculate the electrostatic correction to the bending energy $k_{\mathrm{c}}$, we need to solve the potential up to second order in $u$ since the curvature energy is of the form

$$
F_{\text {bend }}=\frac{1}{4} k_{\mathrm{c}} u^{2} q^{4}
$$

Therefore, expanding the potential in Fourier series, we look for a solution

$\phi(x, z)=\phi_{0}(z)+\alpha^{2} u^{2} \phi_{02}(z)+\alpha u \phi_{1}(z) \cos q x+\alpha^{2} u^{2} \phi_{2}(z) \cos 2 q x+O\left(\alpha^{3} u^{3}\right)$.

Here we define the relevant inverse length $\alpha$ by

$$
\alpha^{2}=\frac{n_{0} e^{2}}{2 \varepsilon T}
$$

We show in Appendix A that $\alpha \sim \pi / 2 d$ in this case, so that $\alpha u$ is a small parameter. We also show in this appendix that a solution of the form given by equation (7) exists, and that this leads to a bending constant $k_{\mathrm{c}} \sim \frac{d}{l} T$ as expected from reference [2]. We have not however calculated the constant of proportionality by this method due to mathematical complexity.

It is desirable to have an estimate of the numerical constant to enable comparison with experiment. We therefore consider the simpler geometry of two membranes curved into concentric cylinders (Fig. 3) with the radius of curvature $R \gg d$. The bending energy is $F_{\text {bend }}=\frac{k_{\mathrm{c}}}{2 R^{2}}$ in this case. The separation of the membranes is $2 d$ as before. There exists a radius between the membranes at which the field is zero, which we will call $R+d_{1}$. The field on the membrane (radius $R$ ) is $\sigma / \varepsilon$. The solution of the Poisson-Boltzmann equation between two cylinders has been given by Fuoss et al. [10]. In Appendix B we apply this to region 1 of figure 3 , again in the highly charged limit where $\lambda \ll d$. To a first approximation the zero field point is midway between the membranes $\left(d_{1}=d\right)$ but when the membranes are curved the boundary shifts slightly toward the centre $\left(d_{1}<d\right)$. It is necessary to calculate $d_{1}$ from the condition that the ion concentration in regions 1 and 2 be continuous. Finally, the bending constant for the curvature of the membrane at radius $R$ is obtained from the energies of the fields in regions 1 and 3 :

$$
k_{\mathrm{c}}=\left(\frac{1}{\pi}-\frac{\pi}{12}\right) \frac{2 d}{\ell} T \sim 0.06 \frac{2 d}{\ell} T, \quad\left(\lambda<d<\kappa^{-1}\right) .
$$

Thus $k_{\mathrm{c}}$ does not depend on the charge density in the limit of high charge density. The 


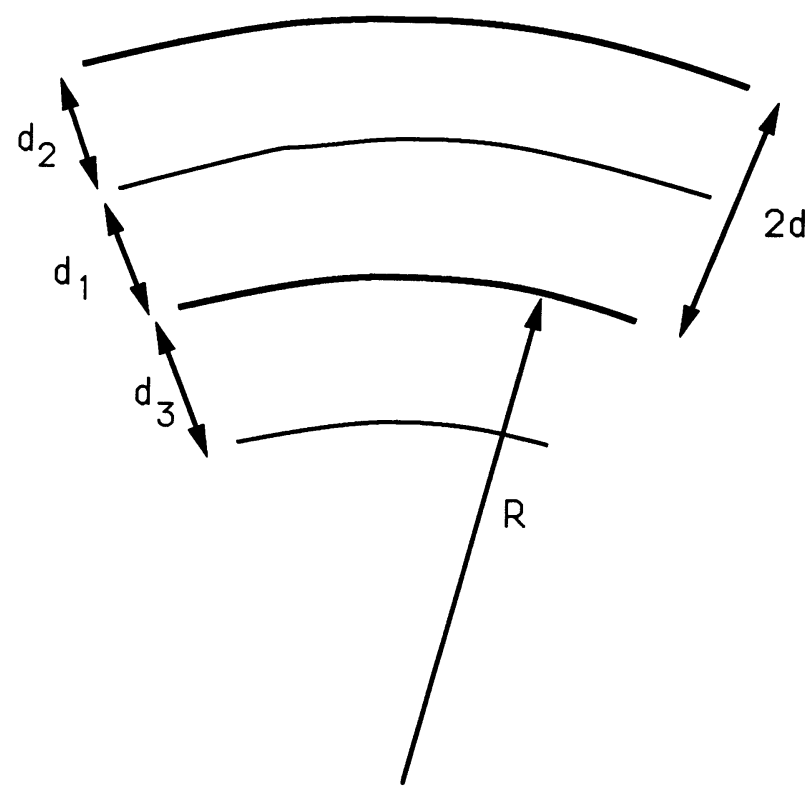

Fig. 3. - Two membranes curved into concentric cylinders. The electric field is zero at radii $R+d_{1}$ and $R-d_{3}$.

numerical constant for the cylindrical geometry gives an idea of the constant for the undulating surface geometry, although it is not bound to be the same. It has already been shown that the same constant is found in the two geometries for the Debye-Hückel region with $\kappa d>1$ [2], and for the Intermediate region (Fogden et al. [11]), but in both cases the problem is independent of $d$, and interactions between neighbouring membranes are unimportant. For the "small $d$ » cases interactions between membranes become important and the bending energy may become dependent on the precise way in which the membrane is curved as discussed below.

Lamellar phases have been studied experimentally by dynamic light scattering (Nallet et al. $[12,13])$ and by synchrotron X ray scattering (Safinya [14], Roux and Safinya [15]). Dilute lamellar phases exist with a repeat distance of several hundred Ångstroms. If such systems are highly charged in the absence of added salt the result (9) implies a $k_{\mathrm{c}} \gg T$, and hence extremely flat, rigid membranes.

\section{Discussion and conclusions.}

The bending constant has now been calculated in all the regions of the diagram of figure 1 except in the ideal gas region where the distance between membranes is small $(d \ll \lambda)$ and the electrostatic interactions are not screened. The bending constant is however known in both regions which border the ideal gas region. The only scaling of the lengths which has a smooth crossover to the known results at the boundaries $\lambda \approx d$ and $\kappa^{2} \lambda d \approx 1$ is that proposed in [2] :

$$
k_{\mathrm{c}}=\frac{\lambda}{\ell} T, \quad\left(d<\lambda<\kappa^{-1}\right) .
$$

The condition $\kappa^{2} \lambda d \approx 1$ was obtained as a boundary for the Debye-Hückel region from the criterion for the validity of the linearised equation - namely $e \phi<T$. This line has another 
physical significance. In both the ideal gas region and the small $d$ Debye-Hückel region the entropy of the ions becomes more important than the electrostatic interactions as $d \rightarrow 0$, and the ions are distributed approximately uniformly between the surfaces. The concentration of counterions is then $\sim \frac{\sigma}{e d}$. From the definitions in section 1 we see that the line $\kappa^{2} \lambda d \approx 1$ is the point at which the counterion concentration equals that of the salt $n_{\infty}$. If we begin in the Debye-Hückel region and gradually reduce the salt concentration the screening length $\kappa^{-1}$ is replaced by $(\lambda d)^{1 / 2}$ in the ideal gas region.

The above calculations suggest several possible experiments. Firstly, it should be possible to see the linear dependence of $k_{\mathrm{c}}$ on $d$ in the Gouy-Chapman region by gradual dilution of the lamellar phase with a solution of fixed, very low salt concentration. Lamellar phases have been found which are stable over a wide range of repeat distances e.g. $50 \AA-800 \AA$ for the sodium dodecyl sulphate systems discussed by Nallet et al. [12]. The linear relationship is limited at large dilutions at the point $d \sim \kappa^{-1}$. There will always be some small concentration of ions in solution, if only from the dissociation of water itself, and therefore $\kappa^{-1}$ is never infinite. At small separations we cross into the ideal gas region at $d \sim \lambda$, where $k_{\mathrm{c}}$ is again independent of $d$. Secondly, we may look for the salt dependence of $k_{\mathrm{c}}$ by gradual addition of salt at a fixed $d$. We expect $k_{\mathrm{c}}$ independent of $n_{\infty}$ until $\kappa^{-1} \sim d$, and then $k_{\mathrm{c}} \sim n_{\infty}^{-1 / 2}$ (from Eq. (3)).

It may also be possible to investigate the dependence on the charge density $\sigma$ by forming membranes of a mixture of charged and uncharged molecules. For small enough $\sigma$ we will be in the ideal gas region $(\lambda>d)$ even for relatively large $d$. Equation (10) presents an apparent problem since it predicts $k \sim 1 / \sigma$ for small $\sigma$. Evidently $k_{\mathrm{c}}$ must tend to zero in the absence of charges. We will of course always arrive at the Debye-Hückel region when $\kappa^{2} \lambda d \sim 1$, when the small salt concentration can no longer be neglected. However this may occur for extremely low charge density, and other effects may occur before then. The typical distance between charges in the membrane is $\sim(e / \sigma)^{1 / 2}$, and when this distance becomes comparable to $d$ we may no longer simply consider a uniformly charged surface but we must introduce explicitely the discreteness of the charges. The calculation of the bending energy has also been done at constant charge density. If the membrane is made by a mixture of charged and uncharged surfactants, the local composition of the membrane fluctuates and these fluctuations are coupled to the undulations of the membrane. We expect this to reduce the electrostatic contribution to the bending energy.

Our solution for the electrostatic field between membranes assumes large wavelength oscillations such that $q<1 / d$ (the expansion of (A11)). For small wavelength oscillations the bending energy is no longer as in equation (6) and no bending constant exists as such. However, the energy for these small oscillations is extremely large and the amplitude of these modes is negligible. The membranes are flat over distances up to the correlation length $\xi$. Using the method of reference [2] we find $\xi \sim d$. Fodgen et al. [10] note the dependence on $q$ for the case of screened interactions, where a bending constant exists only for wavelengths larger than $\kappa^{-1}$. They conclude that for an isolated membrane in the absence of salt the curvature energy is proportional to $u^{2} q^{3}$ and not to $u^{2} q^{4}$, this would be equivalent to a wave vector dependent bending constant $k_{\mathrm{c}} \sim T / q \ell$. We have shown however that in lamellar phases the $u^{2} q^{4}$ form still applies even for no added salt at low wave vectors. This is because the counterion concentration around the membrane remains finite when there is a finite repeat distance between membranes. At higher wave vectors ( $q d \gg 1$ ) all counterions are confined in a distance $q^{-1}$ from the membrane and the bending constant crosses over smoothly to $k_{\mathrm{c}} \sim T / q \ell$.

Finally we wish to consider more precisely the meaning of $k_{\mathrm{c}}$ and $\bar{k}_{\mathrm{c}}$ in the cases where 
$d$ is small. If we take the Debye-Hückel region with $\kappa d<1$, we may also obtain the bending energy in the cylindrical geometry discussed above. The solution may be written in terms of the Bessel functions $I_{0}(\kappa r)$ and $K_{0}(\kappa r)$ as is done for a single cylindrical surface by Winterhalter and Helfrich [3]. The important expansion parameter is $1 / \kappa R$ and not $d / R$. The resulting bending energy is $F_{\text {bend }} \sim \frac{T}{R^{2} \kappa^{3} \ell \lambda^{2}(\kappa d)}$ which is weaker by a factor of $(\kappa d)^{2}$ than the result calculated in the undulating surface geometry (Eq. (2)). In the same region Goldstein et al. [5] calculate the potential between two undulating surfaces at fixed potential rather than at fixed charge density. Expanding their result gives yet a third scaling form for the bending energy. This should be compared to the $\kappa d>1$ case (isolated membranes) where changing the boundary conditions only changes the numerical constant (Kiometzis and Kleinert [9], Bensimon et al. [6]).

Thus for small $\kappa d$ the result depends on the way the membrane is curved and not just the radii of curvature. In this case we consider that the result calculated from the undulating surface geometry is the important one, since it is this which governs the fluctuations in lamellar phases. The cylindrical geometry does not correspond to an experimental situation.

The difference between the two geometries may be interpreted in the following way. When $\kappa d>1$ interactions between the surfaces are screened and negligible, but for $\kappa d<1$ they are important. Curving the surfaces into concentric cylinders does not change the separation of the membranes, whereas for the undulating surfaces there are points closer and further apart than the mean separation. Inter-membrane effects are thus treated in a different fashion in the two geometries, which is very important at small $d$. (We note again that if fluctuations in the charge density are possible, these will be coupled to the curvature of the membrane and will probably affect the bending energy significantly in the small $\kappa d$ case. This point will be addressed in a subsequent paper.) In the ideal gas region there is again a difference between the geometries for the same reason. We expect that equation (10) applies only to the undulating surface geometry. The Gouy-Chapman region is an intermediate case, since here the result is $d$ dependent but the scaling form does not depend on the geometry.

The meaning of the Gaussian curvature constant $\bar{k}_{\mathrm{c}}$ is thus not very clear for these small $d$ regions. In principle an argument of Helfrich [1,3] can be used to calculate $k_{\mathrm{c}}$ directly from the potential between two flat plates (by an integration of the Maxwell stress). This gives a value of $\bar{k}_{\mathrm{c}}$ which scales in the same way as $k_{\mathrm{c}}$ in the Gouy-Chapman region $\left(\bar{k}_{\mathrm{c}} \sim T d / \ell\right)$, but differently in the small $\kappa d$ Debye-Hückel region and the ideal gas region. However $\bar{k}_{\mathrm{c}}$ is only of relevance when comparing the energies of structures of different topologies (vesicles, sponges etc.) and a careful consideration of the electrostatic effects on these structures has yet to be made. The very high bending energies for unscreened charged membranes greatly increase the stability of the lamellar phase relative to other more highly curved structures.

\section{Appendix A.}

Solution for undulating surface geometry.

We first assume that a solution of the Poisson-Boltzmann equation (Eq. (4)) has been obtained and consider the way the free energy may be obtained from this. The free energy is the sum of the electrostatic energy and the contribution from the counterion entropy.

$$
F=\int \mathrm{d} x \int_{u(x)}^{d} \mathrm{~d} z\left(\frac{\varepsilon}{2} \mathbf{E}^{2}+T n \ln \left(n / n^{*}\right)\right)
$$


where $\mathbf{E}=-\nabla \phi$ is the electric field, $n(x, z)$ is the concentration of counterions, which must satisfy $\nabla^{2} \phi=-n e / \varepsilon$, and $n^{*}$ is an arbitrary constant. The distribution of counterions which minimizes $F$ must satisfy $\delta F=0$ for any arbitrary change $\delta n$ about the equilibrium solution. The counterion concentration follows thus a Boltzmann distribution

$$
e \phi+T\left(\ln \left(n / n^{*}\right)+1\right)=0
$$

which leads to the Poisson-Boltzmann equation (4) with $n_{0}=n^{*} e^{-1}$.

We now calculate the change in the free energy caused by a shift $\delta d$ in the membrane position.

$$
\begin{aligned}
\frac{\partial F}{\partial d} \delta d=\int \mathrm{d} x \int_{u(x)}^{d} \mathrm{~d} z\left(\varepsilon \mathbf{E} \cdot \delta E+T \delta n\left(\ln \left(n / n^{*}\right)+1\right)\right)+ \\
+\int \mathrm{d} x \int_{d}^{d+\delta d} \mathrm{~d} z\left(\frac{\varepsilon}{2} E^{2}+T n \ln \left(n / n^{*}\right)\right) .
\end{aligned}
$$

But $\nabla .(\phi \delta E)=e \phi \delta n / \varepsilon-\mathbf{E} . \delta \mathbf{E}$, therefore

$$
\frac{\partial F}{\partial d} \delta d=\int \mathrm{d} x \int_{u(x)}^{d} \mathrm{~d} z[-\varepsilon \nabla \cdot(\phi \delta E)]+\delta d \int \mathrm{d} x \operatorname{Tn}(x, d) \ln \left(n(x, d) / n^{*}\right)
$$

where (A2) has been used. Applying the divergence theorem on the first term and noting that the normal field on the membrane is fixed constant by the boundary conditions we have

$$
\frac{\delta F}{\partial d}=\int \mathrm{d} x\left(\operatorname{Tn}(x, d) \ln \left(n / n^{*}\right)+\varepsilon \phi \frac{\partial E_{z}(x, d)}{\partial z}\right)
$$

and finally

$$
\frac{\partial F}{\partial d}=-\int \mathrm{d} x\left(T n+\varepsilon \phi \frac{\partial E_{x}}{\partial x}\right)
$$

where all the quantities are to be integrated along the midplane between the membranes.

We note that the simpler argument which gives $F=\int_{0}^{\sigma} \phi \mathrm{d} \sigma$ cannot be used here since we have the potential for $\lambda<d$ which is therefore not valid over the whole range of the integral.

We now turn to evaluation of the potential. Defining $y=e \phi / T$ we have

$$
\nabla^{2} y=-2 \alpha^{2} \exp (-y)
$$

We expect a solution

$$
y(x, z)=y_{0}(z)+\alpha^{2} u^{2} y_{02}(z)+\alpha u y_{1}(z) \cos q x+\alpha^{2} u^{2} y_{2}(z) \cos 2 q x \ldots
$$

Substituting into (A4) we have

$$
\begin{aligned}
& \frac{\mathrm{d}^{2} y_{0}}{\mathrm{~d} z^{2}}=-2 \alpha^{2} \exp \left(-y_{0}\right) \\
& \frac{\mathrm{d}^{2} y_{1}}{\mathrm{~d} z^{2}}-2 \alpha^{2} \exp \left(-y_{0}\right) y_{1}=q^{2} y_{1} \\
& \frac{\mathrm{d}^{2} y_{02}}{\mathrm{~d} z^{2}}-2 \alpha^{2} \exp \left(-y_{0}\right) y_{02}=-\frac{\alpha^{2}}{2} \exp \left(-y_{0}\right) y_{1}^{2} .
\end{aligned}
$$


The boundary conditions are $\frac{\partial y}{\partial z}=0$ at $z=d$, and $\nabla y \cdot \overline{\mathbf{n}}=\frac{2}{\lambda}$ at the membrane, where $\overline{\mathbf{n}}$ is a unit vector normal to the surface. Expanding the potential on the membrane as a Taylor series about $z=0$ gives

$$
\begin{aligned}
& \frac{\mathrm{d} y_{0}}{\mathrm{~d} z}=\frac{\mathrm{d} y_{1}}{\mathrm{~d} z}=\frac{\mathrm{d} y_{02}}{\mathrm{~d} z}=0 \\
& \text { at } \quad z=d \\
& \frac{\mathrm{d} y_{0}}{\mathrm{~d} z}=\frac{2}{\lambda} \\
& \text { at } \quad z=0 \\
& \alpha \frac{\mathrm{d} y_{1}}{\mathrm{~d} z}+\frac{\mathrm{d}^{2} y_{0}}{\mathrm{~d} z^{2}}=0 \\
& \text { at } \quad z=0 \\
& \alpha^{2} \frac{\mathrm{d} y_{02}}{\mathrm{~d} z}+\frac{\alpha}{2} \frac{\mathrm{d}^{2} y_{1}}{\mathrm{~d} z^{2}}+\frac{1}{4} \frac{\mathrm{d}^{3} y_{0}}{\mathrm{~d} z^{3}}=\frac{q^{2}}{2}\left(\alpha y_{1}-\frac{1}{\lambda}\right) \quad \text { at } \quad z=0 .
\end{aligned}
$$

The solution for two flat surfaces is

$$
y_{0}=2 \ln (\cos \alpha(z-d))
$$

and from (A6ii) $\alpha$ must satisfy

$$
\tan \alpha d=\frac{1}{\alpha \lambda}
$$

For high charge densities where $\lambda \ll d$ this means

$$
\alpha d=\pi / 2-v
$$

for some small $v$ of order $\lambda / d$. We are interested in the limit $v$ tends to 0 and will show that the bending energy is finite in this limit, even though the electric field diverges at the surface. Putting $y_{0}$ into (A5ii) gives an equation with general solution

$$
y_{1}=A \mathrm{e}^{q(z-d)}(\tan \alpha(z-d)+q)+B \mathrm{e}^{-q(z-d)}(\tan \alpha(z-d)-q) .
$$

Applying the boundary conditions gives

$$
y_{1}=\frac{-2 \sec ^{2} \alpha d\left(\tan \alpha(z-d) \sinh q(z-d)+\frac{q}{\alpha} \cosh q(z-d)\right)}{\left(\sec ^{2} \alpha d+\frac{q^{2}}{\alpha^{2}}\right) \sinh q d+\frac{q}{\alpha} \tan \alpha d \cosh q d} .
$$

Using (A9) in the limit $v \rightarrow 0$ we see that $y_{1}$ on the midplane has the value

$$
y_{1}(d)=-\frac{2 q / \alpha}{\sinh (\pi q / 2 \alpha)} .
$$

We may now evaluate the contribution of the second term in (A3).

$$
-\varepsilon \int \mathrm{d} x \phi \frac{\partial E_{x}}{\partial x}=+\varepsilon \frac{T^{2}}{e^{2}} \int \mathrm{d} x y y^{\prime \prime}=-\varepsilon \frac{T^{2}}{2 e^{2}} \alpha^{2} u^{2} q^{2} y_{1}^{2}(d) \text {. }
$$

Expanding $y_{1}(d)$ in powers of $q$ we find the $u^{2} q^{4}$ term is of order $\varepsilon(T / e)^{2}$ independent of $d$, and by integrating with respect to $d$ the equivalent term in the free energy is $T d / \ell$, as was to be proved. 
The first term of (A3) gives

$$
\begin{aligned}
\int \mathrm{d} x n T & =n_{0} T \exp \left(-y_{0}\right) \int \mathrm{d} x \exp \left(-\alpha u y_{1} \sin q x-\alpha^{2} u^{2} y_{02}-\alpha^{2} u^{2} y_{2} \sin 2 q x\right) \\
& =2 \alpha^{2} \varepsilon \frac{T^{2}}{e^{2}}\left(1-\alpha^{2} u^{2}\left(y_{02}(d)-y_{1}^{2}(d) / 4\right)\right) .
\end{aligned}
$$

To find the $u^{2} q^{4}$ term in (A13) we need the term in $q^{4}$ in $y_{02}$. Expanding $y_{1}$ and $y_{02}$ in powers of $q$, we write

$$
y_{1}=t_{0}+\frac{q^{2}}{\alpha^{2}} t_{1}+\frac{q^{4}}{\alpha^{4}} t_{2}, \quad \text { and } \quad y_{02}=s_{0}+\frac{q^{2}}{\alpha^{2}} s_{1}+\frac{q^{4}}{\alpha^{4}} s_{2},
$$

and substituting into (A5iii) gives

$$
\frac{\mathrm{d}^{2} s_{2}}{\mathrm{~d} \zeta^{2}}-2 \sec ^{2} \zeta s_{2}=g(\zeta)=-\frac{1}{2} \sec ^{2} \zeta\left(2 t_{0} t_{2}+t_{1}^{2}\right)
$$

with $\zeta=\alpha(z-d)$. This can be solved by the method of variation of constants.

$$
\begin{gathered}
s_{2}=A(\zeta)(\zeta \tan \zeta+1)+B(\zeta) \tan \zeta \\
A(\zeta)=\int_{0}^{\zeta}-\tan \zeta g(\zeta) \mathrm{d} \zeta+A^{*} \\
B(\zeta)=\int_{0}^{\zeta}(\zeta \tan \zeta+1) g(\zeta) \mathrm{d} \zeta+B^{*}
\end{gathered}
$$

Since $\frac{\mathrm{d} s_{2}}{\mathrm{~d} z}=0$ at $\zeta=0$ we must have $B^{*}=0$. Therefore $s_{2}(z=d)=A^{*}$, which can be determined from the boundary conditions at $z=0$. We require that $A^{*}$ is a constant (i.e. not a diverging function of $v$ ) when $v$ tends to zero so that all terms in $q^{4}$ in (A13) are independent of $d$. Expanding (A10) in powers of $q$ and evaluating at $z=0$ we find

$$
t_{0} \sim 1 / v, \quad t_{1} \sim v, \quad t_{2} \sim v^{2} .
$$

There is thus no divergence in $t_{1}$ and $t_{2}$. The boundary conditions at $z=0$ for $s_{2}$ are (from (A6iv))

$$
\frac{\mathrm{d} s_{2}}{\mathrm{~d} \zeta}=\frac{t_{1}}{2}-\frac{1}{2} \frac{\mathrm{d}^{2} t_{2}}{\mathrm{~d} \zeta^{2}}
$$

The right hand side can be shown to be $-\sec ^{2} \zeta t_{2}$ which is therefore a constant when $v$ tends to zero. From (A15) we have

$$
\begin{aligned}
\frac{\mathrm{d} s_{2}}{\mathrm{~d} \zeta}=\left(\int_{0}^{\pi / 2-v}-\tan \zeta g(\zeta) \mathrm{d} \zeta\right. & \left.+A^{*}\right) \frac{\mathrm{d}}{\mathrm{d} \zeta}(\zeta \tan \zeta+1)+ \\
& +\left(\int_{0}^{\pi / 2-v}(\zeta \tan \zeta+1) g(\zeta) \mathrm{d} \zeta\right) \frac{\mathrm{d}}{\mathrm{d} \zeta}(\tan \zeta)
\end{aligned}
$$

We now replace all functions in (A17) by their behaviour close to $\zeta=-\pi / 2$. If we evaluate 
(A10) at $\zeta=-\pi / 2+w$ we find that there are divergences in $t_{1}$ and $t_{2}$ which cancel at $w=v$ (i.e. at $z=0$ ) but which contribute to $g(\zeta)$ in the integrals above.

$$
t_{0} \sim 1 / w, \quad t_{1} \sim v / w, \quad t_{2} \sim v / w, \quad(w \neq v) .
$$

Now calculating $g$ we find that the leading term is $\sim \frac{v}{w^{4}}$, hence

$$
\begin{aligned}
\text { const. }=\left[\int_{\pi / 2}^{v} \frac{1}{w}\left(1-\frac{w^{2}}{3}\right) \cdot \frac{v}{w^{4}} \mathrm{~d} w\right. & \left.+A^{*}\right] \frac{\pi}{2 v^{2}}\left(-1-\frac{v^{2}}{3}+O\left(v^{3}\right)\right)+ \\
& +\left[\frac{\pi}{2} \int_{\pi / 2}^{v} \frac{1}{w}\left(1-\frac{w^{2}}{3}+O\left(w^{3}\right)\right) \cdot \frac{v}{w^{4}} \mathrm{~d} w\right] \frac{1}{v^{2}}\left(1+\frac{v^{2}}{3}\right) .
\end{aligned}
$$

The highest order diverging terms all cancel. The highest remaining terms are

$$
\text { const. }=-\frac{\pi}{2 v^{2}} A^{*}+O\left(\frac{1}{v^{2}}\right)+\cdots
$$

from which we see $A^{*}$ must be a constant in order to eliminate the $1 / v^{2}$ divergence. Thus we have demonstrated that all terms in $q^{4}$ in $\frac{\partial F}{\partial d}$ are independent of $d$, and that the bending energy scales as $\frac{d}{l} T$.

\section{Appendix B.}

\section{Solution for cylindrical geometry.}

Setting $y=e \phi / T$ and $\kappa_{1}^{2}=\frac{n_{1} e^{2}}{\varepsilon T}$ the Poisson-Boltzmann equation becomes

$$
\frac{1}{r} \frac{\mathrm{d}}{\mathrm{d} r} r \frac{\mathrm{d} y}{\mathrm{~d} r}=-\kappa_{1}^{2} \exp (-y)
$$

Fuoss et al. [9] give the solution

$$
y=\ln \left(\frac{\kappa_{1}^{2} r^{2}}{2 \beta^{2}} \sin ^{2}(\beta \ln A r)\right)
$$

where $\beta$ and $A$ are arbitrary constants to be determined. The choice of $n_{1}$ is also arbitrary and corresponds to a choice of the zero of the potential. Fuoss et al. choose $n_{1}$ to be the concentration if the counterions were distributed uniformly between the two cylinders, which in region 1 of figure 3 is therefore

$$
n_{1}=\frac{\sigma}{e} \frac{2 R}{\left(R+d_{1}\right)^{2}-R^{2}}=\frac{\sigma}{e d_{1}\left(1+\frac{d_{1}}{2 R}\right)}
$$

Applying the boundary conditions for the field gives

$$
\begin{aligned}
& 1+\beta \cot \left(\beta \ln A\left(R+d_{1}\right)\right)=0 \\
& 1+\beta \cot (\beta \ln A R)=R / \lambda .
\end{aligned}
$$


In the limit $\lambda / d_{1} \rightarrow 0$ we have $\beta d_{1} / R \sim \pi / 2$. We find $\beta$ as an expansion in $d_{1} / R$ and neglect all terms in $\lambda / R$.

$$
\beta=\frac{\pi R}{2 d_{1}}\left(1+\frac{d_{1}}{R}\left(\frac{1}{2}+\frac{4}{\pi^{2}}\right)-\frac{d_{1}^{2}}{R^{2}}\left(\frac{1}{12}+\frac{16}{\pi^{4}}\right) \cdots\right) .
$$

Now the free energy per unit area of side 1 of the membrane is

$$
\begin{aligned}
F_{1} & =\frac{1}{R} \int_{R}^{R+d_{1}} r \mathrm{~d} r\left(\frac{\varepsilon T^{2}}{2 e^{2}}\left(\frac{\mathrm{d} y}{\mathrm{~d} r}\right)^{2}+T n(r) \ln \left(n(r) / n^{*}\right)\right) \\
& =\frac{1}{R} \int_{R}^{R+d_{1}} r \mathrm{~d} r\left(\frac{\varepsilon T^{2}}{e^{2}}\left(\frac{1}{2}\left(\frac{\mathrm{d} y}{\mathrm{~d} r}\right)^{2}+y \nabla^{2} y\right)+T n(r) \ln \left(n_{1} / n^{*}\right)\right) \\
& =\frac{\varepsilon T^{2}}{R e^{2}} \int_{R}^{R+d_{1}} \cdot \mathrm{d} r\left(-\frac{r}{2}\left(\frac{\mathrm{d} y}{\mathrm{~d} r}\right)^{2}+\frac{\mathrm{d}}{\mathrm{d} r}\left(r y \frac{\mathrm{d} y}{\mathrm{~d} r}\right)\right)+\frac{\sigma}{e} T \ln \left(n_{1} / n^{*}\right) \\
& =-\frac{\varepsilon T^{2}}{2 \operatorname{Re}^{2}} \int_{R}^{R+d_{1}} \mathrm{~d} r r\left(\frac{\mathrm{d} y}{\mathrm{~d} r}\right)^{2}+\frac{\sigma T}{\varepsilon}\left(-y(R)+\ln \left(n_{1} / n^{*}\right)\right) .
\end{aligned}
$$

All terms in $d_{1} / R$ cancel from the final two terms above, therefore we are left with the integral. Setting $\xi=\beta \ln A R$ gives

$$
\begin{aligned}
F_{1}=\frac{\varepsilon T^{2}}{R e^{2}} \int_{\beta \ln A R}^{\beta \ln A\left(R+d_{1}\right)} \frac{2 \mathrm{~d} \xi}{\beta}(1+\beta \cot \xi)^{2} & + \text { const. }= \\
& =\frac{\varepsilon T^{2}}{e^{2}}\left\{\frac{\pi^{2}}{2 d_{1}}+\frac{d_{1}}{R^{2}}\left(\frac{8}{\pi^{2}}-\frac{\pi^{2}}{24}\right)-\frac{4}{R} \ln d_{1}\right\} .
\end{aligned}
$$

We now have to calculate $d_{1}, d_{2}$ and $d_{3}$. The value of $\beta$ in the region 3 is calculated in the same way as for region 1 , and differs from equation (B2) by a change in the sign of the second term :

$$
\beta_{3}=\frac{\pi R}{2 d_{3}}\left(1-\frac{d_{3}}{R}\left(\frac{1}{2}+\frac{4}{\pi^{2}}\right)-\frac{d_{3}^{2}}{R^{2}}\left(\frac{1}{12}+\frac{16}{\pi^{4}}\right) \cdots\right)
$$

from which

$$
\beta_{2}=\frac{\pi(R+2 d)}{2 d_{2}}\left(1-\frac{d_{2}}{R+2 d}\left(\frac{1}{2}+\frac{4}{\pi^{2}}\right)-\frac{d_{2}^{2}}{(R+2 d)^{2}}\left(\frac{1}{12}+\frac{16}{\pi^{4}}\right)\right) .
$$

The ion concentration must be continuous at the point $R+d_{1}$, i.e. $n\left(R+d_{1}\right)=n\left((R+2 d)-d_{2}\right)$. This is satisfied if $\beta_{1}=\beta_{2}$. From this we obtain

$$
\begin{aligned}
& d_{1}=d\left(1-C d / R+C d^{2} / R^{2} \ldots\right), \quad C=\frac{1}{2}-\frac{4}{\pi^{2}} \\
& d_{3}=d\left(1+C d / R+C d^{2} / R^{2} \ldots\right) .
\end{aligned}
$$

The total free energy per unit area of the membrane is

$$
F=F_{1}+F_{3}=\frac{\varepsilon T^{2}}{e^{2}}\left\{\frac{\pi^{2}}{2}\left(\frac{1}{d_{1}}+\frac{1}{d_{3}}\right)+\frac{d_{1}+d_{3}}{R}\left(\frac{8}{\pi^{2}}-\frac{\pi^{2}}{24}\right)-\frac{4}{R} \ln \frac{d_{1}}{d_{3}}\right\} .
$$


The bending energy is the sum of all the terms in $1 / R^{2}$ in this expression :

$$
F_{\text {bend }}=\frac{\varepsilon T^{2} d}{e^{2} R^{2}}\left(4-\frac{\pi^{2}}{3}\right)
$$

from which $k_{\mathrm{c}}$ is as given in equation (9).

The potential on the outer membrane surface (region 1) differs slightly from that on the inner surface (region 3 ). There is a potential difference across the membrane $\Delta \phi \sim \frac{T d}{e R}$. For the assumption of the independence of the two sides of the membrane to be valid the energy stored in the field inside the membrane (which is also $O\left(1 / R^{2}\right)$ ) must be small compared to the bending energy.

$$
t \varepsilon_{\mathrm{L}}\left(\frac{\Delta \phi}{t}\right)^{2} \ll \frac{\mathrm{d} T}{\ell R^{2}}
$$

Hence $t \gg \frac{\varepsilon_{\mathrm{L}}}{\varepsilon} d$, as given in section 2. The numerical value of the bending constant is therefore a function of $\varepsilon_{\mathrm{L}}$ and $t$ and we have only calculated one extreme.

\section{Acknowledgements.}

We thank D. Andelman and P. Pincus for their continued interest, and D. Roux for useful suggestions. P. G. Higgs acknowledges the support of the Commission of the European Communities under contract no. SC1000263.

\section{References}

[1] Helfrich W. (1981) Physics of Defects, Ed. R. Balian, Les Houches session XXXV, 1980 (North Holland).

[2] Pincus P., Joanny J.-F., Andelman D., Europhys. Lett. 11 (1990) 763.

[3] Winterhalter M., Helfrich W., J. Phys. Chem. 92 (1988) 6865.

[4] Duplantier B., Goldstein R. E., Pesci A. I., Romero-Rochin V. (1990) preprint.

[5] Goldstein R. E., Pesci A. I., Romero-Rochin, V., Phys. Rev. A (1990).

[6] Bensimon D., David F., Leibler S., Pumir A., J. Phys. France 51 (1990) 689.

[7] Mitchell D. J., Ninham B. W., Langmuir 5 (1989) 1121.

[8] LekKerkerker H. N. W., Physica A 159 (1989) 319.

[9] Kiometzis M., Kleinert H., Physica A 140 (1989) 520.

[10] Fuoss R. M., Katchalsky A., LifSon S., Proc. Nat. Acad. Sci. 37 (1951) 579.

[11] Fogden A., Mitchell D. J., NinhaM B. W., Langmuir 6 (1990) 159.

[12] Nallet F., Roux D., Prost J., J. Phys. France 503147.

[13] Nallet F., Roux D., Prost J., Phys. Rev. Lett. 62276.

[14] Safinya C. R., Proceedings of Geilo school, Soft Condensed Matter Physics, Eds. T. Riste, D. Sherrington (Plenum) (1989).

[15] Roux D., Safinya C. R., J. Phys. France 49 (1988) 307. 